\title{
Heterogeneous energy landscapes of individual luminescent conjugated polymers
}

\author{
Jun-Jih Liang, ${ }_{1,2, *}$ J. D. White, ${ }^{1,3}$ Y. C. Chen, ${ }^{1,4}$ C. F. Wang, ${ }^{1,4}$ J. C. Hsiang, ${ }^{1,4}$ T. S. Lim, ${ }^{1}$ W. Y. Sun, ${ }^{1}$ J. H. Hsu, ${ }^{1,5}$ \\ C. P. Hsu, ${ }^{6}$ M. Hayashi, ${ }^{7}$ W. S. Fann, ${ }^{1,4, \dagger}$ K. Y. Peng, ${ }^{8}$ and S. A. Chen ${ }^{8}$ \\ ${ }^{1}$ Institute of Atomic and Molecular Sciences, Academia Sinica, P.O. Box 23-166, Taipei, Taiwan \\ ${ }^{2}$ Department of Physics, Fu-Jen University, Xinzhuang, Taiwan \\ ${ }^{3}$ Department of Electrical Engineering, Yuan Ze University, Chungli, Taiwan \\ ${ }^{4}$ Department of Physics, National Taiwan University, Taipei, Taiwan \\ ${ }^{5}$ Department Material Science and Optoelectronic Engineering, National Sun Yat-sen University, Kaohsiung, Taiwan \\ ${ }^{6}$ Institute of Chemistry, Academia Sinica, Taipei, Taiwan \\ ${ }^{7}$ Center for Condensed Matter Sciences, National Taiwan University, Taipei, Taiwan \\ ${ }^{8}$ Department of Chemical Engineering, National Tsing Hua University, Hsinchu, Taiwan
}

(Received 8 May 2006; published 22 August 2006)

\begin{abstract}
The energy landscape of single, isolated, short- and long-chain luminescent conjugated polymers was studied by photobleaching spectral shift analysis and interphoton time measurements. It has been found that the energy landscape of a polymer is dependent on both the conjugation length distribution as well as the arrangement of these conjugated segments. Energy funnels responsible for abrupt drops in the photoluminescence time trace intensity are found to be wide (in terms of the number of absorbing segments feeding energy into them) rather than deep (in terms of energy relative to the other emission sites in the polymer). Experimental results indicate that the energy landscape is influenced, but not dictated, by the solvent polarity. Moreover, it was observed that spectral blueshifts do not always accompany the quenching of a funnel, pointing to the existence of multiple independent regions of approximately the same energy distribution within an individual polymer.
\end{abstract}

DOI: 10.1103/PhysRevB.74.085209 PACS number(s): 78.55.Kz, 36.20. - r, 78.66.Qn, 82.35. -x

\section{INTRODUCTION}

The energy landscape of macromolecules is not only a topic of considerable concern within the polymer community but also has implications for biomolecular studies such as that of proteins. ${ }^{1}$ Luminescent conjugated polymers provide good model systems to investigate this issue as there is no need to attach external chromophores as probes and they can be prepared with different chain lengths and conformations for controlled experiments. The heterogeneity of the energy landscape, such as whether it depends on the dimension of polymer (short chain versus long chain) or the solvents used to prepare the polymer samples, can thus be addressed experimentally. In practice, the interplay between energy landscape and energy transfer is an important issue in the design of light emitting diodes and solar cells using organic semiconductors.

Considerable effort has been made to study energy transfer within a polymer. Ultrafast spectroscopy has been used to monitor how energy funnels from absorption to emission segments on the sub-ps to ps time scale. At slightly longer time scales, time-resolved experiments record the ensemble behavior of the emitters as the equilibrium emission excitons ensemble. Single-molecule spectroscopy is complementary to the ensemble measurements of ultrafast spectroscopy. While single-molecule experiments due to their limited time resolution cannot observe the early relaxation processes probed by ultrafast spectroscopy, they can, however, monitor the subsequent behavior of emission excitons on an individual polymer basis. In other words, the events follow excitons settling into the bottom of the energy funnels. Monitoring the single molecule at long time scale can thus tell us what events occurred at an earlier time. For example, if not all the energy funnels into a single emitting site but rather goes into two sites, then two emitters will be observed in the single-molecule experiment.

In single-molecule experiments, considerable effort has been devoted to the study of excitation energy migration within a conjugated polymer in relation to its conformation. ${ }^{2-5}$ Experimental and theoretical results indicate that energy transfer along the polymer backbone (intrachain energy transfer) is slower than three-dimensional interchain energy transfer ${ }^{6-9}$ Conformation has been shown to be dependent on preparation as well as on local environment. Yu et $a l^{3}$ and Hollars et al. ${ }^{10}$ have shown that MEH-PPV (poly[2-methoxy, 5-(2'-ethyl-hexyloxy)-p-phenylene-vinylene]) in the nonpolar solvent toluene folds into a tight structure with only a few emission excitons in an individual polymer. In general, nonpolar solvents or a high molecular weight host matrix yield tightly coiled conformation, whereas polar solvents or a low molecular weight host matrix lead to a more extended conformation, ${ }^{5,11}$ supporting a large number of emission excitons. ${ }^{10}$ Previously, we demonstrated by single-molecule polarization experiments that short, defect-free polymers were rod shaped ${ }^{12}$ and that energy transfer in such a conjugated polymer was inefficient by comparing the experimentally observed spectral fluctuation with that calculated from a molecular exciton model. ${ }^{6}$

While considerable work has been done in dealing with energy transfer issues, much less work has been done relating to the energy landscape and characterization of energy funnels. The distribution of the light emitting segments or chromophores is dependent on both the conjugated length distribution and the arrangement of these conjugated segments. The latter is strongly dependent on the conformation of the polymer. Combined, these determine the energy landscape. Regarding the former, numerous models have been 
proposed dealing with the conjugated length distribution within a polymer. While these models are generally not applicable for the discussion of dynamics involving coherence on the femtosecond time scale, ${ }^{13}$ they have proven to be useful in helping to understand the absorption and photoluminescence (PL) emission of isolated polymers in solution. ${ }^{6}$ One such representative model is the relatively simple molecular exciton model of Chang et al. ${ }^{14}$ in which a long polymer is composed of a number of oligomers of different conjugation lengths.

It is important to distinguish between absorption and emission excitons. The absorption spectrum reflects the distribution of the absorption excitons, whereas the PL spectrum reflects the distribution of the emission excitons. Alternatively, one can say that the average conjugation length of the absorbing segments (ACLA) is reflected by the absorption spectrum, while the average conjugation length of emitting segments (ACLE) is seen in the PL spectrum. In the case of no exciton migration, absorption and emission occur at the same conjugated segment (oligomer) and thus ACLE =ACLA. In the opposite extreme, the ACLE will reflect the conjugation length of a single emitting exciton having no relation to the ACLA of the polymer. Because photobleaching refers to the quench of the emission excitons, the PL time trace reveals the number of emission excitons in a polymer chains and the evolution of the ACLE of the polymer. The observation of multiple steps in this trace shows that there are many emission excitons. While the size of the steps in the PL time trace indirectly provides information on the number of absorption excitons transferring energy to a given emitting exciton, unfortunately in single-molecule experiments, it is not easy to take absorption spectra and thus we have no direct information about absorption excitons and ACLA in an individual polymer.

In this work, we shall study the energy landscape of single, isolated polymers, exploring photophysical conformation changes from different origins such as chain length and solvent polarity based on changes in the ACLE. To this end, we developed a method, which we call the single-molecule spectral shift analysis, to analyze changes in the PL of a polymer under constant excitation. This allows a quantitative comparison of ACLE for single polymers in different conformations and its change during photobleaching. Measurements of ACLE along with photon correlation measurements combined with insights from a distribution of conjugation lengths model allow us to develop a more thorough picture of the energy landscape within a single polymer and the effects processing has on this landscape.

\section{EXPERIMENT}

\section{A. Sample preparation}

Two extensively studied PPV derivatives, MEH-PPV and DOO-PPV [poly(2,5-dioctyloxyp-phenylene-vinylene], were investigated in the current study. The first was chosen as its solubility in a variety of solvents allows solvent effects on conformation to be investigated. The second was chosen as its limited solubility enables polymers of different chain lengths to be extracted and studied. ${ }^{6}$ Pristine MEH-PPV
$\left(M_{w}=500 \mathrm{kDa}, \sim 1800\right.$ monomers) was dissolved in either (1) toluene, (2) mixed toluene-chloroform with a volume ratio 1:4, or (3) chloroform in order to compare the effects of solvent on conformation. ${ }^{5}$ The solution was diluted into a polystyrene solution and spin-cast onto a fused silica substrate. A buffer layer of poly(ethylene oxide) (PEO) was spun on the sample to prevent the subsequently evaporated Al cap layer (deposited to limit exposure to oxygen) from quenching the PL. Rodlike DOO-PPV $\left(M_{n}=8.2 \mathrm{kDa}, \sim 24\right.$ monomers $^{6}$ ) was dissolved in chloroform, diluted into a polystyrene solution, spin-cast onto a substrate, and sealed in a dry nitrogen box. ${ }^{6}$ For comparison purposes, a thin polystyrene film containing isolated DiI (1, 1' -dioctadecyl$3,3,3^{\prime}, 3^{\prime}$-tetramethylindocarbocyanine perchlorate) molecules was also prepared and protected from oxygen exposure by an Al cap layer.

\section{B. Optical system}

Photoluminescence under constant intensity illumination (Ar-ion laser, $\lambda=488 \mathrm{~nm}$ ) was collected in epi-flourescence mode. The double-port technique, where a polarization insensitive beamsplitter $\left(\lambda_{\text {center }}=555 \mathrm{~nm}\right)$ splits the PL into two channels to be detected by calibrated avalanche photodiodes (APDs) operating in single-photon counting mode, allowed spectral information to be recorded with a time resolution of $1 \mathrm{~ms}$ for the DiI molecules and $10 \mathrm{~ms}$ for PPV polymers. (The rapid spectral shift in the first milliseconds of the time trace for a single molecule would not be observable if the whole spectrum were being recorded because of the required integration time for adequate signal to noise ratio.) A detailed description of the experimental setup has been reported elsewhere. ${ }^{6}$

In order to determine the number of simultaneously independent emitters in the long-chain MEH-PPV single polymer, interphoton time measurements were carried out using the Hanbury-Brown and Twiss coincidence setup ${ }^{15}$ with femtosecond laser. The excitation wavelength of $445 \mathrm{~nm}$ was obtained from a frequency-doubled mode-locked tunable Ti:sapphire laser (Coherent, Mira 900) operating at a repetition rate of $76 \mathrm{MHz}$. The time between the arrival of photons at the two detection APDs were recorded by a time-correlated single-photon counting (TCSPC) module (Becker \& Hickl $\mathrm{GmbH}, \mathrm{SPC}-600)$.

\section{Spectral shift analysis technique}

The PL spectrum reflects the ACLE of the polymer. Changes in the PL spectrum reflect changes in the ACLE. For efficient migration of thermalized excitons, energy is likely to funnel to the longest segment, where emission takes place. Consequently, during photobleaching, one expects a gradual shortening of ACLE due to the fact that the emitting site is vulnerable as it spends greater time in the excited state than its shorter segment counterparts. This shortening will be reflected by shifting of the PL spectrum towards shorter wavelengths as intensity declines. On the contrary, in the absence of exciton migration, there is no preferential quenching site and thus one expects the ACLE and hence spectrum to vary randomly during photobleaching. This change in 
ACLE can be evaluated in terms of the spectral change as intensity drops. By calculating the spectral shift defined as ${ }^{6}$

$$
S(t)=\frac{I_{>}(t)-I_{<}(t)}{I_{>}(t)+I_{<}(t)},
$$

where $I_{>}$and $I_{<}$denote the intensities registered by the longand short-wavelength channel, respectively, the evolution of the spectral change can be obtained with high temporal resolution. To allow a histogram of many polymers to be compiled, it is necessary to shift the origin. Based on the fact that during photobleaching, the PL intensity of a single molecule decreases as a result of the active excitons being quenched, a reference $\left(S_{\mathrm{o}}\right)$ can then be set at the point where PL intensity is maximal. The spectral shift relative to the spectral shift at maximum intensity is thus given by

$$
S_{r}(t)=S(t)-S_{0} .
$$

A spectrum that undergoes blueshift (redshift) will be characterized by negative (positive) values of $S_{r}$ reflecting a shortening (lengthening) of ACLE during photobleaching. The average of the relative spectral shift, $\left\langle S_{r}(t)\right\rangle$, is expected to be $\sim 0$ for a polymer in which there is no change in ACLE due to a lack of thermalized exciton migration during the polymers survival time. Calculations based on the molecular exciton theory show that a change of $\sim 0.11$ in $S_{r}$ reflects a lengthening of the ACLE of the polymer by a single monomer. ${ }^{14}$

A key advantage of $S_{r}$ is that it can be summed for a number of individual polymers (note that this is quite different from an ensemble experiment) and a histogram constructed of the probability of a molecule exhibiting a given spectral shift. Polymers can then be categorized according to solvent, lifetime, type of intensity trace, embedded matrix, or any other experimental parameter and conclusions drawn about changes in ACLE and thermalized exciton transport within a type of polymer during photobleaching. The slope of $S_{r},\left(d S_{r} / d t\right)$, allows the change in ACLE at a particular point in a polymer's lifetime to be evaluated. $d S_{r} / d t<0$ implies ACLE is shortening while emission sites are being quenched, whereas $d S_{r} / d t \sim 0$ implies no change in ACLE is occurring as the polymer is quenched.

\section{RESULTS}

\section{A. Photoluminescence time trace during photobleaching}

Figure 1 compares the PL time trace for a small molecule (DiI), rodlike short-chain DOO-PPV polymer, and two longchain MEH-PPV polymers. The small molecule's time trace [Fig. 1(a)] exhibits the typical single chromophore characteristic of on-off blinking, ${ }^{2}$ as the molecule enters into and leaves reversible dark states. For rodlike short-chain DOOPPV [Fig. 1(b)], intensity changes are discrete rather than gradual and the emission occurs at several intermediate intensity levels. Figures 1(c) and 1(d) display the time traces of two long-chain polymers. Although prepared similarly, the time trace in Fig. 1(c) exhibits pronounced, abrupt intensity fluctuations accompanied by gradual intensity decline, while that in Fig. 1(d) is best described as a monotonic decrease in

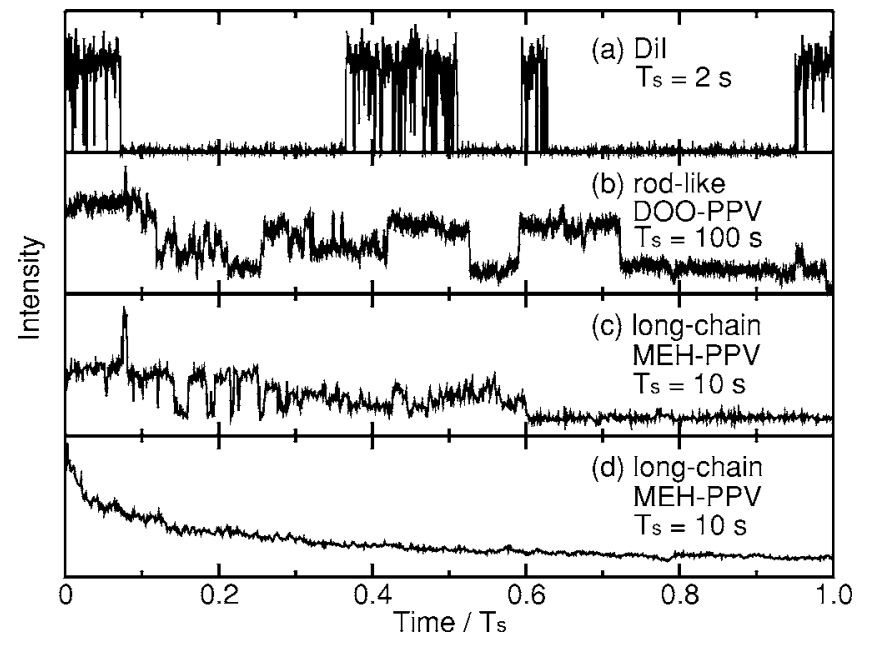

FIG. 1. Comparison of the PL time traces behavior for (a) DiI, (b) rodlike DOO-PPV, and (c) and (d) long-chain MEH-PPV/ toluene. To emphasize the qualitatively different behavior, only a portion of each trace is presented and neither intensity nor temporal span $\left(T_{\mathrm{s}}\right)$, are displayed using the same scale.

intensity with occasional slight increases in intensity. The majority of long-chain polymers exhibit behavior that is a combination of that seen in Figs. 1(c) and 1(d). The great variety of time trace behavior seen in long-chain polymers stems from the fact that the chain length is much longer than its persistent length, allowing chains the freedom to fold into a variety of three-dimensional structures. ${ }^{4}$ The exact conformation is determined by each individual's inhomogeneous environment. ${ }^{5,11}$ This conformation determines the migration of thermalized excitons from tens to hundreds of absorption sites to the emission sites. In contrast, the length of the shortchain polymer supports only a few absorption excitons and its rodlike nature limits the number of possible conformations, resulting in much more well-defined behavior. ${ }^{12}$ The qualitative similarity of the time trace of the long-chain polymer in Fig. 1(c) with the rodlike polymer is striking when one considers that their relative absorption cross sections and hence PL emission intensities differ by a few orders of magnitude.

\section{B. Spectral shift evolution}

Photoluminescence time traces were taken and the spectral shift calculated for 43 rodlike and 301 long-chain polymers (72 toluene, 180 toluene-chloroform, and 49 chloroform). Figure 2(a) shows typical time traces recorded by the two APDs for a long-chain polymer along with the calculated relative spectral shift $\left(S_{r}\right)$ [Fig. 2(b)]. The solid line is the 101-point running average of $S_{r}$ showing the tendency of the spectral shift. $S_{r}$ falls from 0 to -0.31 ( $72 \%$ of the final value) in the first $35 \mathrm{~s}$, reaches -0.37 near $t=70 \mathrm{~s}$, and approaches -0.43 at about $t=140 \mathrm{~s}$. It stays around this value for another $520 \mathrm{~s}$ (not shown). This trace is typical of the majority of long-chain polymers in that $d S_{r} / d t$ is initially negative and at later times tends towards zero or fluctuates between positive and negative values. This is true irrespective of whether there are large discrete intensity fluctuations 


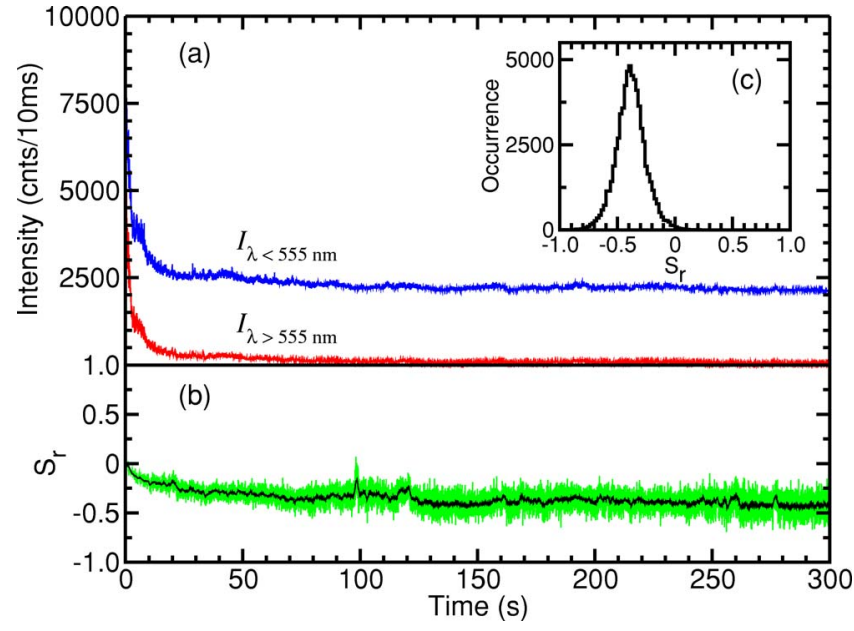

FIG. 2. (Color online) (a) Typical PL time trace of MEH-PPV/ toluene recorded by the two channels. $I_{\lambda<555 \mathrm{~nm}}$ is offset vertically for clarity. (b) $S_{r}$ as a function of time. The green (gray) line is calculated by Eq. (2). The black (dark gray) line is the time average of $1 \mathrm{~s}$, indicating the tendency of the spectral shift. (c) Histogram of the spectral shift of (b).

in the time trace. The histogram of $S_{r}$ [Fig. 2(c)] has a mean, determined by Gaussian fitting, of -0.37 . This represents a shortening of the ACLE of the polymer during photobleaching of $\sim 3$ monomer units as a result of the longer conjugated segments being quenched first. The fact that $d S_{r} / d t \sim 0$ in the later phases of the polymer survival time reflects the isolated nature of the remaining unbleached shorter conjugated segments.

Figure 3 shows the temporal evolution of the mean of the ensemble averaged spectral shift $\left(\left\langle S_{r}\right\rangle_{\mathrm{ens}}\right)$ for all measured long-chain polymers. At the beginning of photodegradation $\left(t<0.1 t_{\mathrm{s}}\right.$ where $t_{\mathrm{s}}$ is the survival time of the polymer), $d\left\langle S_{r}\right\rangle_{\mathrm{ens}} / d t$ is strongly negative reflecting a rapid shortening of the ACLE as the polymer is photobleached. Under the assumption that the probability of a quench event is related

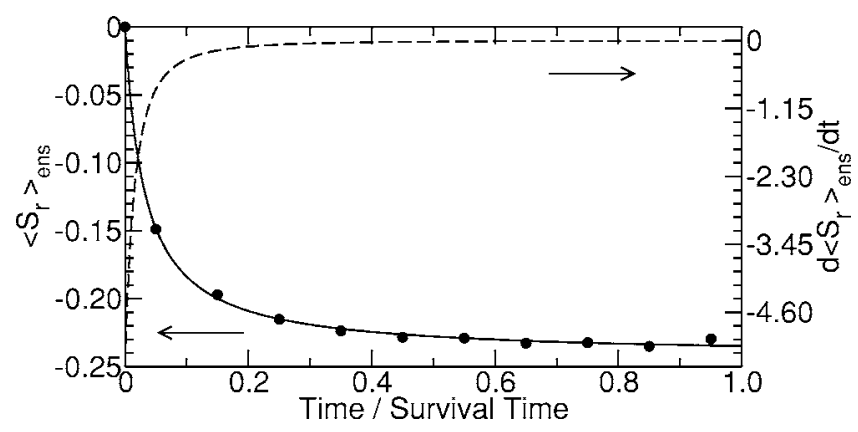

FIG. 3. Temporal evolution of the mean of the ensemble average of $S_{r}$ for 301 long-chain polymers prepared in a variety of solvents. The survival time of each polymer was partitioned into ten equal segments. An area normalized histogram of $S_{r}$ calculated from the two PL time traces was constructed for each segment. The mean, $\left\langle S_{r}\right\rangle_{\text {ens }}$, for each segment is represented by the solid circles. The solid line is obtained by a least squares curve fitting to $\sim 1 /(1$ $+a t)$ with $a$ as a variable parameter. $d\left\langle S_{r}\right\rangle_{\text {ens }} / d t$ is plotted as a dashed line. to the probability of an oligomer being excited, this is consistent with the view that the emitting segments with the longest conjugation lengths are first photobleached due to the fact that they spend more time in the excited state. Sections with longer conjugation length are expected to spend more time in the excited state as a result of (i) a more favorable absorption cross section at the excitation wavelength (the molecular exciton theory of Chang et al. ${ }^{14}$ predicts that segments of the polymer conjugated over $<4$ monomers have limited absorption at the excitation frequency) and possibly (ii) migration of thermalized excitons to these sections either along the chain backbone or via energy funnels. ${ }^{3}$ As these long conjugated segments are quenched, the slope becomes gradually flatter, reaching $\sim 0$ for $t \geqslant 0.6 t_{s}$ indicating that there is no further shortening of the ACLE during the final $40 \%$ of the polymer's survival time. As the polymer photobleaches, the increasingly segmented nature of the polymer as emission sites are quenched results in the single longchain polymer becoming more like a collection of isolated rodlike short-chain polymers that also experience no change in ACLE during photobleaching [as will be discussed in the next section with reference to Fig. 4(c)]. As it is not expected to be efficient thermalized exciton migration between such sections, and the remaining emitting excitons have a relatively shorter conjugation length and hence smaller absorption cross section at the excitation wavelength, these excitons spend relatively little time in the excited state. They are thus not easily photobleached and emit at a low intensity for a long time.

\section{Categorization of polymers}

In the following sections, relative spectral shift histograms are constructed by summing relative spectral shifts of individual polymers after the polymers have been divided according to a number of mutually exclusive criteria.

\section{Categorization by chain length}

Figure 4 compares the relative spectral shift for rodlike short-chain and long-chain polymers. In Figs. 4(a) and 4(b) the histograms for average value of relative spectral shift $\left(\left\langle S_{r}\right\rangle\right)$ for individual polymers are compared. Nearly half (22 polymers) of the 43 measured rodlike polymers show almost no spectral shift $\left(\left|\left\langle S_{r}\right\rangle\right|<0.05\right)$ and only three have $\left|\left\langle S_{r}\right\rangle\right|$ $>0.15$. In contrast, $89 \%$ of the long-chain polymers $(269 / 301)$ exhibit blue spectral shift $\left(\left\langle S_{r}\right\rangle<-0.05\right)$. In $20 / 301$ polymers there was little or no spectral shift and only $6 / 301$ polymers show strong redshifts $\left(\left\langle S_{r}\right\rangle>0.15\right)$. In Fig. $4(\mathrm{c})$, the spectral shift histograms of individual polymers are summed. While the full-widths at half-maximum (FWHM) of the summed histograms are similar $(0.30$ and 0.46 for short-chain and long-chain, respectively), the long-chain polymers histograms is relatively symmetric while that for the short chain is quite asymmetric. In addition, the center of the histogram for long-chain MEH-PPV $(-0.23)$ and for rodlike DOO-PPV $(-0.013)$ differs greatly. The negative value for the long-chain polymer indicates that the average conjugation length of emitting segments shortens by $\sim 2.5$ mono- 

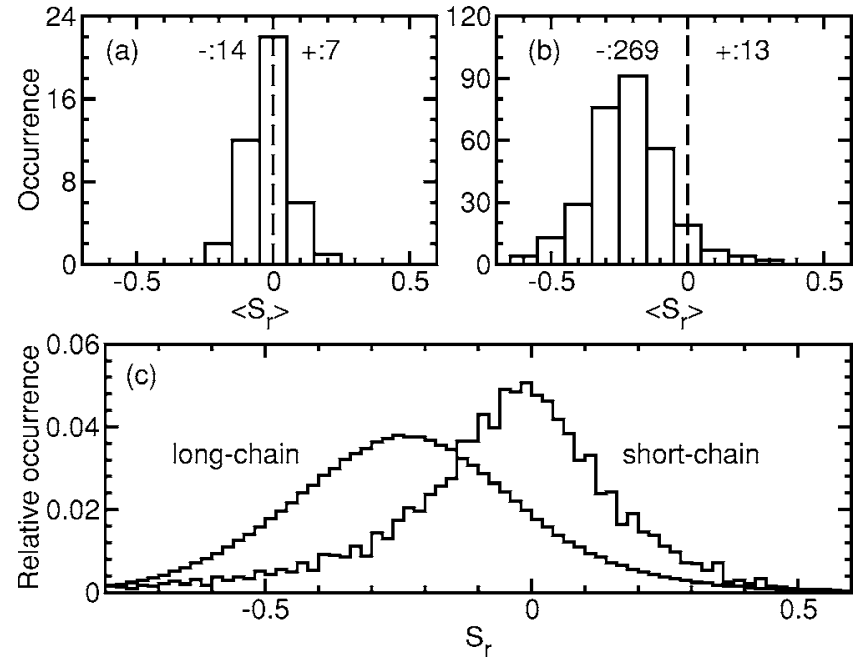

FIG. 4. Comparison of the relative spectral shift for rodlike short-chain and long-chain polymers. Histograms of $\left\langle S_{r}\right\rangle$ for (a) short-chain and (b) long-chain polymers. (c) Area normalized ensemble histograms of $S_{r} ; 43$ short-chain polymers and 301 longchain polymers are analyzed.

mers during photodegradation. The negligible shift for the rodlike polymer indicates that the average conjugation length of the polymer does not change. For the case of short-chain polymers the effect of a quench event is to darken a complete conjugated segment rather than to shorten its conjugation length. This result is in agreement with our previous study in which the efficiency of thermalized exciton migration in rodlike DOO-PPV was studied by the molecular exciton model, ${ }^{14}$ and demonstrated to be inefficient. ${ }^{6}$ The blueshift observed for the long-chain polymer suggests the existence of efficient exciton migration in at least sections of these polymers in agreement with much previous work..$^{3-5}$ The tail emerging in the negative $S_{r}$ in the spectral shift histogram for short-chain polymers [Fig. 4(c)] is due both to an undersampling of the conjugation length distribution and to defect induced conformational change. While the majority of the short-chain polymers are defect-free, defects in a small minority allow for a more collapsed structure and thus efficient energy transfer.

\section{Categorization by solvent}

Figure 5 compares the relative spectral shift obtained for long-chain polymers processed with different solvents. In Figs. 5(a)-5(c) the histograms for average value of relative spectral shift $\left(\left\langle S_{r}\right\rangle\right)$ for individual polymers are compared. $83 \%(60 / 72)$ of the polymers dissolved in toluene, $93 \%$ $(169 / 180)$ of the polymers dissolved in the mixed solvent, and $82 \%(40 / 49)$ of the polymers dissolved in chloroform exhibit blue spectral shift $\left(\left\langle S_{r}\right\rangle<-0.05\right)$. In all three solvents, a strong redshift $\left(\left\langle S_{r}\right\rangle>0.15\right)$ is rarely observed: $1 \%(1 / 72)$ for toluene, $2 \%(3 / 180)$ for toluene-chloroform, and $4 \%$ $(2 / 49)$ for chloroform. The change in ACLE of the polymer during photobleaching is thus relatively unaffected by solvent.

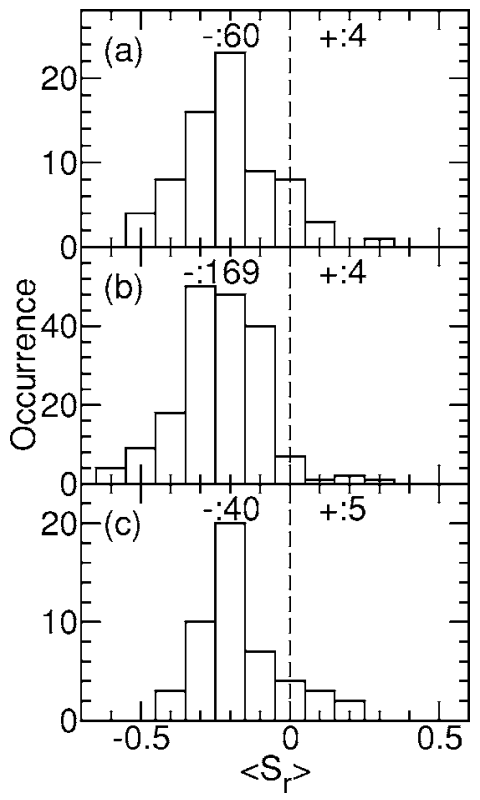

FIG. 5. Comparison of the relative spectral shift obtained for long-chain polymers processed with different solvents. Histograms of $\left\langle S_{r}\right\rangle$ for the polymer dissolved in (a) toluene, (b) mixed toluenechloroform, and (c) chloroform.

In Fig. 6, the spectral shift histograms of individual polymers are summed. The summed histograms are well fit by a single Gaussian with peaks at $-0.23(\sigma=0.21)$ and -0.20 $(\sigma=0.19)$ for toluene and chloroform, respectively. In contrast to the large difference between rodlike and long-chain polymers, the solvent induced change in the center of the summed histogram is relatively small. While the relative values are consistent with the view that the polymer in a nonpolar solvent is more compact, one would expect that the choice of a nonpolar (toluene) or polar (chloroform) solvent in processing would have a much greater effect on the change in ACLE during photobleaching. In any case, the fact

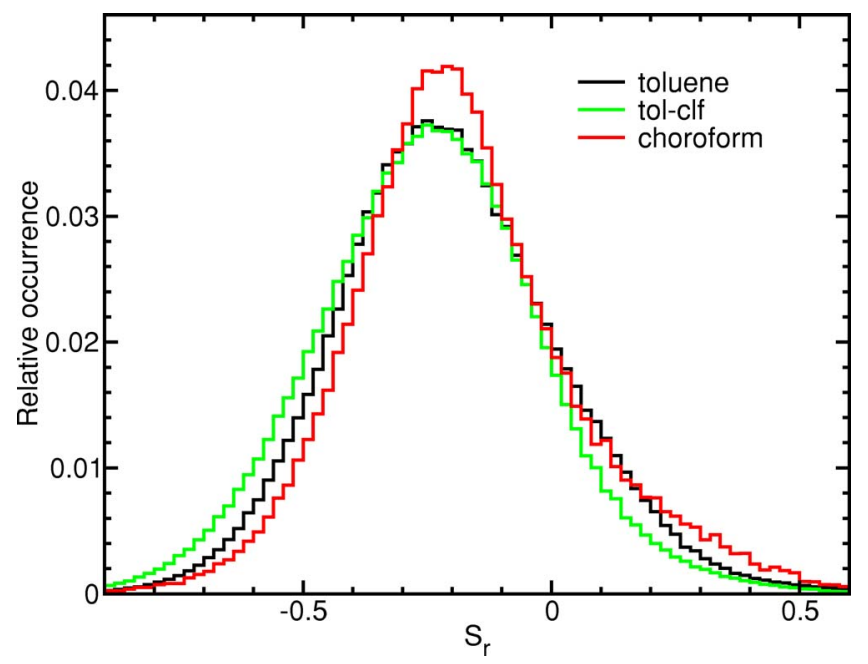

FIG. 6. (Color online) Area normalized ensemble histograms of $S_{r} ; 72$ polymers were processed in toluene (black; dark gray), 180 in the mixed solvent (green; light gray), and 49 in chloroform (red; gray). 


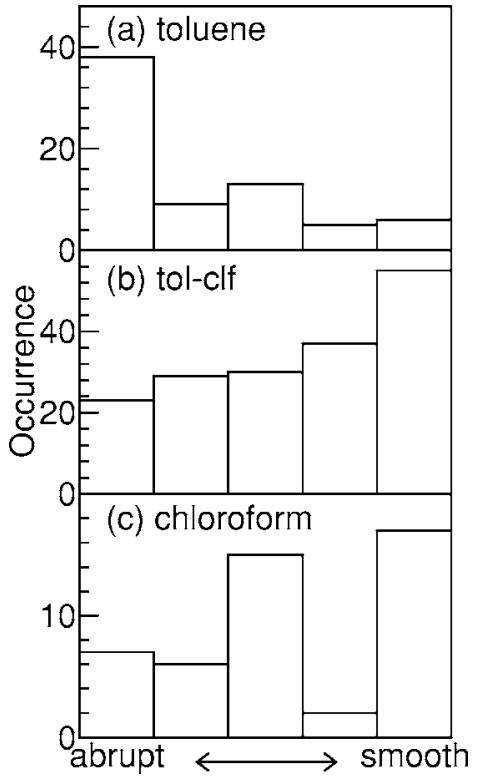

FIG. 7. Histogram of the various time trace behavior for longchain MEH-PPV prepared by dissolving in (a) toluene, (b) toluenechloroform, and (c) chloroform; 72 polymers were processed in toluene, 180 in the mixed solvent, and 49 in chloroform.

that the center of the summed histograms are separated by less than $\sigma_{\mathrm{av}} / 6\left[\sigma_{\mathrm{av}}=0.207, \Delta S_{r}(\right.$ center $\left.)=0.034\right]$ clearly indicates that the choice of solvent is not a key factor influencing the change in ACLE during photobleaching.

\section{Categorization by time trace characteristics}

Figures 1(c) and 1(d) show that both abrupt and monotonic decline in PL intensity during photobleaching can occur in long-chain samples prepared with the same solvent. In order to study the significance of the different timedependent PL intensity behaviors, time traces of the longchain polymers were divided into five categories of abrupt, semiabrupt, intermediate, semismooth, and smooth behavior. While the sorting of individual time trace into a definite group between two adjacent categories is not exact, the classification of the two end groups of abrupt and smooth behaviors is unambiguous. Figure 7 summarizes the distribution of the five categories for long-chain polymers prepared by toluene, mixed toluene-chloroform, and chloroform, respectively. For toluene prepared samples, the majority of the polymers show abrupt behavior [Fig. 7(a)]. Still, there are certain numbers of polymers displaying behaviors of the other groups. Similarly, the mixed toluene-chloroform prepared polymers display time trace behavior in all five categories with a tendency towards the smooth behavior [Fig. 7(b)]. Figure 7(c) shows that while a considerable number of polymers exhibit smooth behavior, a large variety of behaviors is also seen in chloroform prepared polymers. In summary, polymers prepared by every solvent exhibit all five behaviors of time traces. PL time traces are generally considered to be direct evidence of polymer conformation. ${ }^{4}$ These results suggest that solvent polarity influences but does not determine the conformation of long-chain polymers.

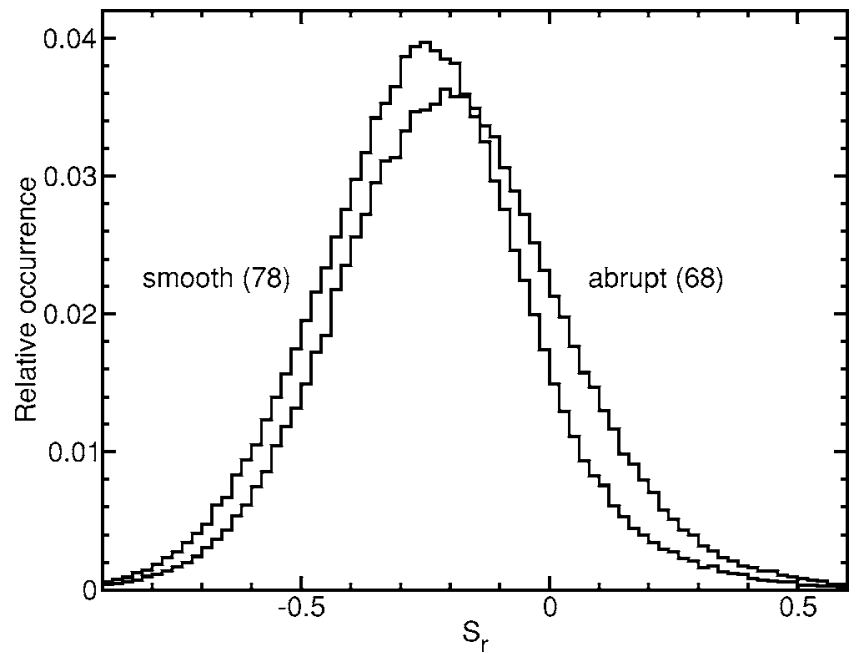

FIG. 8. Area normalized histograms of $S_{r}$ for time traces exhibiting abrupt (68 polymers) and smooth (78 polymers) behavior.

As in Figs. 4 and 5 the spectral shift histograms of individual polymers falling into different categories can be summed and compared. The histograms for the two extreme groups are shown in Fig. 8. Both histograms are symmetric and fitted by a single Gaussian. The center of the histogram for the 78 polymers exhibiting the most smooth behavior is at -0.25 with $\sigma=0.20$. On the opposite extreme, the center of the histogram for 68 polymers exhibiting the most abrupt behavior is -0.20 with $\sigma=0.22$. The difference in peak position $\left[\Delta S_{r}\right.$ (center) $]$ between the two histograms is $\sim \sigma / 4$. Polymers characterized by a smooth decreasing time trace exhibit, on the average, a slightly larger spectral shift than those whose decay is dominated by abrupt intensity changes. This reflects a slightly greater change in ACLE of those polymers whose decay is gradual than those whose decay is abrupt ( $\sim 2.2$ monomers versus $\sim 1.8$ monomers). This result is quite surprising, as a time trace dominated by abrupt steps is seen as an evidence of a more coiled than extended structure. ${ }^{5}$ In that picture, migration of thermalized excitons in such a tightly coiled conformation is expected to take place more preferentially than in a looser structure resulting in a larger blue spectral shift-the opposite of what is observed in this experiment.

In order to get more insight into this apparently perplexing behavior, it is necessary to look at individual polymers. For polymers whose PL time trace is characterized by a smooth decrease in intensity (such as that shown in Fig. 2), the spectrum almost invariably exhibits a gradual blue spectral shift indicating a rather smooth decrease in the ACLE. Segments having a longer conjugation length bleach first due to their large absorption cross section at the excitation wavelength and possibly migration of thermalized excitons to these sites. For polymers whose time traces are characterized by abrupt drops in intensities, generally attributed to energy funnels being quenched, one expects a sudden blue spectral shift as has been reported in previous work with MEH-PPV. ${ }^{3}$ However, as we look at the data we find not only sudden blue spectral shifts but also red and even no spectral shift accompanying abrupt intensity drops. These three types of behavior 


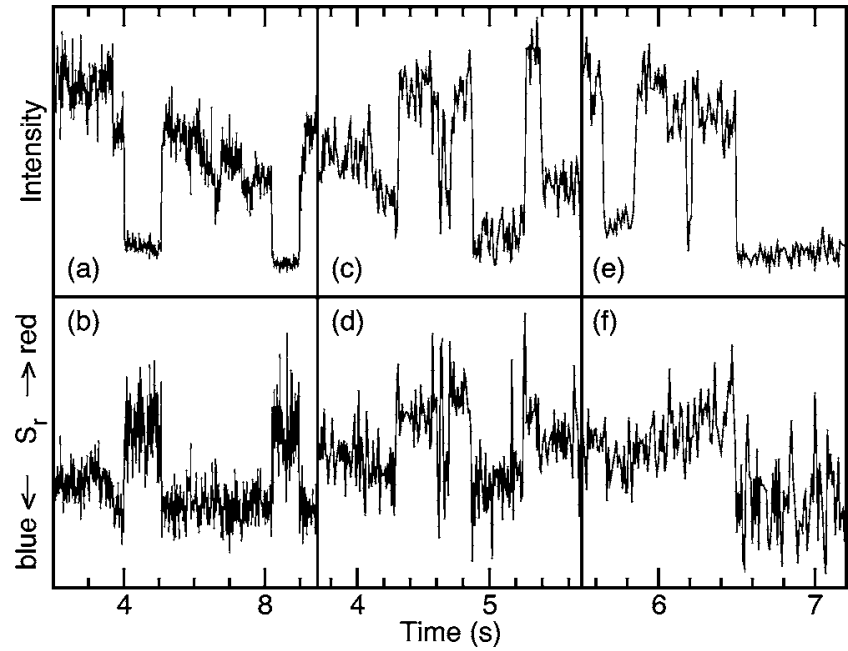

FIG. 9. Spectral shifts accompanying abrupt drops in the PL intensity time trace in single long-chain polymers. (a) and (b) intensity drops at $t=4.0 \mathrm{~s}$ and $8.2 \mathrm{~s}$ are accompanied by a redshift. (c) and (d) intensity drops at $t=4.9 \mathrm{~s}$ and $5.4 \mathrm{~s}$ are accompanied by a blueshift. (e) and (f) intensity drops at $t=5.6 \mathrm{~s}$ and $6.2 \mathrm{~s}$ are independent of any spectral shift.

are shown in Fig. 9. The left panes [Figs. 9(a) and 9(b)] show a polymer in which the PL spectrum undergoes redshift when the intensity drops $(t \sim 4.0 \mathrm{~s}$ and $8.2 \mathrm{~s})$. The middle panes [Figs. 9(c) and 9(d)] show a polymer in which the PL spectrum undergoes blueshift when the intensity drops $(t \sim 4.9 \mathrm{~s}$ and $5.4 \mathrm{~s}$ ). The right panes [Fig. 9(e) and 9(f)] show a polymer in which the first and second intensity drops $(t \sim 5.6 \mathrm{~s}$ and $6.2 \mathrm{~s}$ ) are unaccompanied by a spectral shift. The diverse behaviors observed suggest that the model of a polymer dominated by intensity drops as a loose extended chain and single core ${ }^{16}$ or energy funnel ${ }^{3,4}$ may require some additional refinement. Qualitatively, this type of spectral behavior is very similar to that observed for short-chain polymer. ${ }^{6}$ In that paper, this behavior was used as an evidence of inefficient migration of thermalized excitons along the polymer backbone with emission coming from individual, isolated, conjugated segments. The key difference between the two is that the intensity of the PL differs by orders of magnitude. This suggests that, while the same explanation will not suffice in this case, there may be only a few independent emitters present in these long chains.

\section{Intensity correlation measurements}

Interphoton arrival time measurements were performed to study the number of simultaneously independent emitters in the single long-chain MEH-PPV polymers. Typical time traces and the corresponding interphoton arrival time distributions of the smooth and the abrupt categories for samples prepared by chloroform are shown in Fig. 10 and Fig. 11, respectively. In the case of the smoothly decreasing PL time trace [Fig. 10(a)], the ratio of the peak at zero time offset to that at finite offset is 1 [Fig. 10(b)]. This lack of photon antibunching indicates that there are a large number of uncorrelated, simultaneously active, emitters in this single
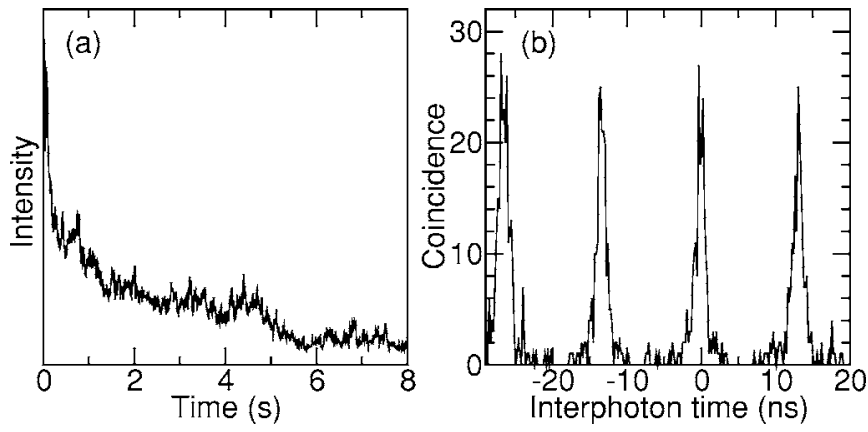

FIG. 10. (a) Photoluminescence intensity time trace and (b) interphoton arrival time distribution for a polymer exhibiting smooth behavior.

polymer. In contrast, for the time trace exhibiting abrupt intensity drops [Fig. 11(a)], the ratio of the peak at zero time offset to that at finite offset [Fig. 11(b)] is 50\%. Usually, such intensity correlation measurements are used to deduce the average number of the independent emitters using the ratio of the number of photon pairs in the central peak to the average number of coincidences in the neighboring lateral peaks. ${ }^{15}$ While it would be tempting to conclude that on average two independent chromophores are active in this single polymer, no such conclusion can be made as the above derivation is based on the assumption that the emitters are of approximately equal strength-which is clearly not the case in this experiment. What can be said is that, on average, over the survival time of the polymer, approximately $50 \%$ of the emission is from one emitter with a limited number of weaker emitters making up the remainder of the emission. Along with the steps shown in the time trace [Fig. 11(a)], this suggests that an emitting exciton at the bottom of a funnel or in a core region exhibits blinking while emitting excitons in other core regions and outlying areas are gradually photobleached. There appears to be little communication between different regions.

\section{DISCUSSION AND CONCLUSIONS}

For the rodlike short-chain polymers, the lack of change in ACLE during photobleaching results in a picture of the polymer as a collection of relatively independent emission
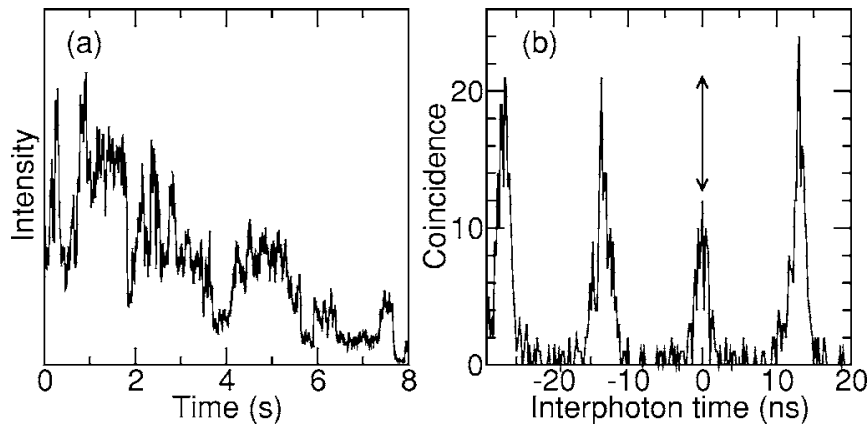

FIG. 11. (a) Photoluminescence intensity time trace and (b) interphoton arrival time distribution for a polymer whose time trace is dominated by abrupt changes in intensity behavior. 
sites coupled by relatively slow thermalized exciton migration. This picture is in agreement with Wang et al. ${ }^{6}$

For the longer polymers, the picture one obtains from the literature suggests more diverse structure with solvent playing a dominate role in determining conformation. ${ }^{5}$ For polymers in which the solvent is nonpolar (toluene), MEH-PPV takes on a collapsed, folded conformation in which energy funnels rapidly from several hundred absorption sites or chromophore units ${ }^{3,4}$ to a few low transition energy traps $^{3-5,10}$ before emission. In contrast for the less studied case of polymers deposited from a polar solvent (chloroform), Huser et al. ${ }^{5}$ have developed a picture of a polymer in an extended conformation in which the single polymer chain behaves as a large ensemble of independent luminescent centers. Sun et al. ${ }^{16}$ presented a more general picture of a polymer in which a core region (many absorbers, single emitter) coexists with extended regions (many absorbers and emitters).

The current work in which spectral shift is used to follow the change of ACLE of the polymer coupled with the presentation of single-molecule (not averaged) photon correlation results for MEH-PPV enables us to put additional flesh on the picture. In particular, three key points arise out of the present work, as follows:

(i) Solvent choice influences but does not dictate conformation and photophysical behavior;

(ii) Energy funnels are not deep relative to the distribution of conjugation lengths within a polymer; and

(iii) Multiple noninteracting core or collapsed regions exist within a single polymer. In the following paragraphs we will discuss these points in turn.

\section{A. Solvent choice influences but does not dictate conformation}

The first point comes out of the analysis associated with Figs. 5-7. Figure 5 shows that there is little difference in the change in ACLE during photobleaching for polymers formed from polar or nonpolar solvents. The analysis associated with Fig. 7 shows while 53\% (38/72) exhibit the characteristics of a collapsed folded conformation expected for a nonpolar solvent, it is by no means $100 \%$. Similarly for the polar solvent, while 35\% (17/49) exhibit the characteristics of a completely extended conformation without any energy funnels, characteristics of a more collapsed conformation, are seen in $65 \%$. Clearly the choice of solvent influences but does not dictate polymer conformation.

\section{B. Energy funnels are not deep relative to the distribution of conjugation lengths}

The second point follows from the analysis of the average spectral shift experienced over the lifetime of the polymer (Fig. 8). While the FWHM of each of the two groups is quite wide, the average spectral shift for those polymers exhibiting smooth behavior (i.e., extended conformation) is -0.25 , while that for those exhibiting the most abrupt behavior (i.e., collapsed conformation) is -0.20 . The implication is that energy funnels are not deep relative to the range of conjugation lengths in the extended polymer. This suggests that rather than creating a few low-transition energy traps, the effect of the collapsed conformation is to allow excitons to migrate efficiently through three-dimensional channels to the longest existing conjugation length segment in that region of the polymer. The fact that these segments are not longer than the longest segments in extended regions is reasonable, as one would expect that the extended regions of the polymer are generally straighter and have fewer defects (leading to longer conjugation length) then the collapsed regions.

\section{Multiple noninteracting regions exist in a single polymer}

The third point arises out of the variety of spectral shifts observed in the case of abrupt intensity drops (Fig. 9). In the picture of the polymer with a single collapsed region or core, the photobleaching of this region is expected to result in a sudden blue spectral shift ${ }^{16}$ as has been observed in previous work. ${ }^{3}$ This result suggests that rather than a single core region, multiple photophysically independent collapsed or core regions in which the excitation energy of one region has a rather small tendency to migrate to another region are present in the polymer. Within each core region, energy funnels from multiple absorbing segments into lower energy conjugated segment where photoemission occurs. Once this segment is photobleached, there is no contribution to emission from this region. Generally, cores with the largest number of absorbing excitons will photobleach first as the lowest energy conjugated segment spends a greater time in the excited state. However, there is no necessary correspondence between the number of absorbing excitons and length of the longest conjugated segment in a collapsed region. In addition, a site with a higher probability of photobleaching does not necessarily bleach first. Such a multiple core picture of the polymer is supported by the photon correlation measurements (Fig. 11) which indicate that for chains exhibiting the abrupt PL time traces associated with a collapsed structure, there is a limited number of emitting segments, most likely more than two but fewer than ten [Fig. 11(b)].

\section{Relation to molecular exciton model and comments on energy migration}

These results provide support for the molecular exciton model of Chang et al., ${ }^{14}$ where a polymer is considered as a collection of oligomers of different conjugation lengths and thus distinct absorption and PL spectra. In this model, the relative frequency of the occurrence of oligomers of different conjugation lengths is determined experimentally by fitting the absorption and emission spectra in solution. Between absorption and emission, excitons are free to migrate. In applying the model to MEH-PPV, it follows that (i) The addition of one monomer to an oligomer results in a spectral shift of $\sim 0.11$ to the red. (ii) The shortest oligomers (i.e., $N \leqslant 4$ ) are rarely directly excited by our laser as they have a very small absorption cross section at the excitation wavelength $(\lambda$ $=488 \mathrm{~nm}$ ). (iii) The conjugation lengths of oligomers excitable by our laser have a range of $\sim 5$ monomer units. Thus, the maximum spectral shift (i.e., all excitons but except those absorbed by $N=4$ oligomers migrate to the longest conjugated segment before emission) is $S_{r}<-0.55$ during photobleaching. Our experimental observations are consistent 
with this model. Firstly, the values of $S_{r}\left(S_{r} \sim-0.20\right)$ measured experimentally are in the range predicted by the model without the need of introducing any new species or traps. Secondly, it follows from the model that since short conjugation length oligomers $(N \leqslant 4)$ are rarely excited, they do not easily photobleach. The PL time trace should thus have an extremely long flat tail. This is what is seen experimentally (Fig. 2 for a single polymer and in Fig. 3 where $d S_{r} / d t \sim 0$ at long time).

This idea of a distribution of conjugation lengths is quite helpful as an aid in understanding the origin of the differing energy landscapes. The conformation of the polymer determines the degrees of freedom for exciton migration. In an extended polymer, an exciton excited at a given point has a choice of two directions in which to migrate- either left or right along the energy backbone. Thus there will be a number of local minimum separated by short conjugated segments which serve to slow or block the migration. The result is a PL time trace that decays gradually. In the short-chain case, in which there are only $\sim 4$ oligomers, there are even fewer degrees for freedom and migration is more easily blocked, with excitons absorbed on either of the two end oligomers having only one possible direction of movement. The result is steplike decay in the PL time trace, few emitters, and little exciton migration. In a polymer with core or collapsed regions, the exciton has much more freedom of movement within each of these cores, as an exciton is not restricted to one-dimensional travel and can therefore bypass short oligomers to find a minimum. The result is similar to the shortchain case: steplike decay in the PL time trace and few emitters.

\section{ACKNOWLEDGMENTS}

This research is supported by National Nanoscience and Nanotechnology Project (Grant No. 93-2120-M-002-009) and MOE Program for Promoting Academia Excellence of Universities under Grant No. 91-E-FA04-4A. J.-J. Liang thanks the National Science Council of R.O.C. for financial support.
*Corresponding author.

Electronic address: phys2021@mails.fju.edu.tw

† Corresponding author.

Electronic address: fann@gate.sinica.edu.tw

${ }^{1}$ H. Frauenfelder, A. R. Bishop, A. Garcia, A. Perelson, P. Schuster, D. Sherrington, and P. J. Swart, Landscape Paradigms in Physics and Biology: Concepts, Structures, and Dynamics (North-Holland, Amsterdam, 1997).

${ }^{2}$ W. T. Yip, D. Hu, J. Yu, D. A. Vanden Bout, and P. F. Barbara, J. Phys. Chem. A 102, 7564 (1998).

${ }^{3}$ J. Yu, D. Hu, and P. F. Barbara, Science 289, 1327 (2000).

${ }^{4}$ D. Hu, J. Yu, K. Wong, B. Bagchi, P. J. Rossky, and P. F. Barbara, Nature 405, 1030 (2000).

${ }^{5}$ T. Huser, M. Yan, and L. J. Rothberg, Proc. Natl. Acad. Sci. U.S.A. 97, 1187 (2000).

${ }^{6}$ C. F. Wang, J. D. White, T. L. Lim, J. H. Hsu, S. C. Yang, W. S. Fann, K. Y. Peng, and S. A. Chen, Phys. Rev. B 67, 035202 (2003).

${ }^{7}$ D. Beljonne, G. Pourtois, C. Silva, E. Hennebicq, L. M. Herz, R. H. Friend, G. D. Scholes, S. Setayesh, K. Müllen, and J. L. Brédas, Proc. Natl. Acad. Sci. U.S.A. 99, 10982 (2002).
${ }^{8}$ T. Q. Nguyen, J. Wu, V. Doan, B. J. Schwartz, and S. H. Tolbert, Science 288, 652 (2000).

${ }^{9}$ E. Hennebicq, G. Pourtois, G. D. Scholes, L. M. Herz, D. M. Russell, C. Silva, S. Setayesh, A. C. Grimsdale, K. Mullen, J. L. Bredas, and D. Beljonne, J. Am. Chem. Soc. 127, 4744 (2005).

${ }^{10}$ C. W. Hollars, S. M. Lane, and T. Huser, Chem. Phys. Lett. 370, 393 (2003)

${ }^{11}$ S. S. Satori, S. De Feyter, J. Hofkens, M. Van der Auweraer, and F. De Schryver, Macromolecules 36, 500 (2003).

${ }^{12}$ J. D. White, J. H. Hsu, Shu-Chun Yang, Wunshain Fann, G. Y. Pern, and S. A. Chen, Chem. Phys. Lett. 338, 263 (2001).

${ }^{13}$ X. Yang, T. E. Dykstra, and G. D. Scholes, Phys. Rev. B 71, 045203 (2005).

${ }^{14}$ R. Chang, J. H. Hsu, W. S. Fann, K. K. Liang, C. H. Chang, M. Hayashi, J. Yu, S. H. Lin, E. C. Chang, K. R. Chuang, and S. A. Chen, Chem. Phys. Lett. 317, 142 (2000).

${ }^{15}$ K. D. Weston, M. Dyck, P. Tinnefeld, C. Müller, D. P. Herten, and M. Sauer, Anal. Chem. 74, 5342 (2002).

${ }^{16}$ W. Y. Sun, S. C. Yang, J. D. White, J. H. Hsu, K. Y. Peng, S. A. Chen, and W. S. Fann, Macromolecules 38, 2966 (2005). 